\title{
Association of circulating long non-coding RNA MALAT1 in diagnosis, disease surveillance, and prognosis of acute ischemic stroke
}

\author{
Hongbo Ren (i) ${ }^{1}$, Feng Wu(i) ${ }^{1}$, Bin Liu (i) ${ }^{1}$, Zhiyuan Song (1) ${ }^{1}$, and Dacheng Qu (i) ${ }^{2}$ \\ ${ }^{1}$ Department of Neurosurgery, HanDan Central Hospital, Handan, China \\ ${ }^{2}$ Department of Neurosurgery, Hebei University of Engineering, Handan, China
}

\begin{abstract}
We aimed to investigate the association of long non-coding RNA metastasis-associated lung adenocarcinoma transcript 1 (IncMALAT1) with acute ischemic stroke (AIS), and its association with disease severity, inflammation, and recurrence-free survival (RFS) in AIS patients. One hundred and twenty AIS patients and 120 controls were recruited. Venous blood samples from AIS patients (within $24 \mathrm{~h}$ after symptoms onset) and controls (at entry to study) were collected to detect plasma Inc-MALAT1 expression by real-time quantitative polymerase chain reaction. AIS severity was assessed by the National Institutes of Health Stroke Scale (NIHSS) score. Plasma concentrations of inflammation factors (including C-reactive protein (CRP), tumor necrosis factor $\alpha$ (TNF- $\alpha$ ), interleukin (IL)-6, IL-8, IL-10, IL-17, and IL-22) were measured and RFS was calculated. Inc-MALAT1 expression was decreased in AIS patients compared to controls, and it had a close correlation with AIS (AUC $=0.791,95 \%$ CI: 0.735-0.846). For disease condition, Inc-MALAT1 expression negatively correlated with NIHSS score and pro-inflammatory factor expression (including CRP, TNF- $\alpha$, IL-6, IL-8, and IL-22), while it positively correlated with anti-inflammatory factor IL-10 expression. Furthermore, Inc-MALAT1 expression was elevated in AIS patients with diabetes. For prognosis, no statistical correlation of Inc-MALAT1 expression with RFS was found, while a trend for longer RFS was observed in patients with IncMALAT1 high expression compared to those with Inc-MALAT1 low expression.
\end{abstract}

Key words: Acute ischemic stroke; IncRNA MALAT1; Inflammation; Survival; Disease severity

\section{Introduction}

Acute ischemic stroke (AIS), a common disorder caused by embolic or thromboembolic occlusion in an artery supplying the brain, results in irreversible infarction of brain tissue and functional impairment, which causes economic and social burden for families (1-3). Current treatments of AIS focus mainly on recovering cerebral circulation and increasing the likelihood of independent living following AIS. Several restrictions exist in current therapies (such as narrow treatment time window and low recanalization rate), and the prognosis of AIS is still far from satisfactory (4-6). Increasing studies disclose that early identification of AIS risk and prediction of prognosis may help improve treatment outcomes of AIS patients $(7,8)$. Thus, searching for convincing biomarkers that monitor AIS risk and predict prognosis is urgently needed.

Long non-coding RNAs (IncRNAs) are RNA transcripts more than 200 nucleotides and rarely encode proteins, which play essential roles in regulation of protein-coding genes and signaling pathways related to development of diseases (8). IncRNA metastasis-associated lung adenocarcinoma transcript 1 (Inc-MALAT1), located on human chromosome 11q13.1, is abundantly expressed and an evolutionarily conserved IncRNA throughout a variety of mammalian species (7). Accumulating data from basic and clinical studies have disclosed that Inc-MALAT1 could protect ischemia-induced brain microvascular endothelial cells (BMECs), implying that Inc-MALAT1 might be involved in cerebrovascular pathologies in stroke $(9,10)$. Meanwhile, Inc-MALAT1 has been reported to be downregulated and primarily play an anti-inflammatory role in patients with neurological disease as well as with cardiovascular and cerebrovascular disease $(11,12)$. Considering the participation of Inc-MALAT1 in cerebrovascular pathologies of stroke and its anti-inflammation effect on cardiovascular and cerebrovascular diseases, we hypothesized that Inc-MALAT1 might serve as a biomarker for disease risk and progression in AIS via regulating inflammation level, whereas related evidence is seldom 
reported. Hence, we conducted this study to investigate the correlation of Inc-MALAT1 expression with AIS, and the association of Inc-MALAT1 expression with disease severity, inflammation level, as well as recurrence-free survival (RFS) in AIS patients.

\section{Material and Methods}

\section{Participants}

In total, 120 AIS patients and 120 controls with high stroke risk factors were recruited from our hospital between July 2014 and June 2017. For the AIS patients, screening criteria were as follows: i) primary diagnosis of AIS by clinical presentation, brain non-contrast computed tomography (CT), or diffusion-weighted magnetic resonance imaging, according to the "Guidelines for the Early Management of Patients with Acute Ischemic Stroke: A Guideline for Healthcare Professionals from the American Heart Association/American Stroke Association (AHA/ASA)"(13); ii) more than 18 years of age; iii) admitted to our hospital within $24 \mathrm{~h}$ after symptom onset; and iv) able to be regularly followed-up. The exclusion criteria were: i) evidence of intracranial hemorrhage; ii) treated with immunosuppressant within 1 month; iii) suffered from infection (active or in the preceding 14 days of stroke); iv) accompanied by hematological malignancies or solid tumors; and v) women who were pregnant or nursing. As for the controls, all of them had no history of stroke or malignancies and were presented with at least three stroke risk factors, such as hypertension, diabetes mellitus, hyperlipidemia, smoking, etc. The Institutional Review Board of HanDan Central Hospital (China) approved the study protocol, and all participants or their guardians provided written informed consent.

\section{Data collection and stroke severity assessment}

At entry to the study, baseline data of AIS patients were obtained through interviews and medical records, including age, gender, body mass index (BMI), smoking, hypertension, diabetes mellitus, hyperlipidemia, hyperuricemia, and chronic kidney disease (CKD). Hypertension was defined as a history of high blood pressure $(\geqslant 140 / 90 \mathrm{mmHg}$ ) reported by the respondent or current use of antihypertensive medication; diabetes mellitus was defined as a previous diagnosis, treatment with insulin or oral hypoglycemic medications, fasting plasma glucose $\geqslant 126 \mathrm{mg} / \mathrm{dL}$, or glycosylated hemoglobin $\geqslant 6.5 \%$; hyperlipidemia was defined as current use of antilipidemic medication, total cholesterol $\geqslant 5.70 \mathrm{mmol} / \mathrm{L}$, serum triglycerides $\geqslant 1.70 \mathrm{mmol} / \mathrm{L}$, or low-density lipoprotein cholesterol $\geqslant 3.10 \mathrm{mmol} / \mathrm{L}$; hyperuricemia was defined as serum uric acid $\geqslant 420 \mu \mathrm{mol} / \mathrm{L}$ for men and $\geqslant 360 \mu \mathrm{mol} / \mathrm{L}$ for women $(14,15)$. Severity of AIS was assessed within the day of admission by use of the National Institutes of Health Stroke Scale (NIHSS) score (according to the
Guidelines for the Early Management of Patients With Acute Ischemic Stroke) (13). The NIHSS score aimed at assessing neurological impairment ranging from 0 to 42, a higher score indicates more serious nerve damage, and the classification of severity was as follows: 0-1 point, normal or near normal; 2-4 points, mild stroke; 5-15 points, moderate stroke; $16-20$ points, moderatesevere stroke; 21-42 points, severe stroke. Besides, basic characteristics of controls including age, gender, BMI, smoking, hypertension, diabetes mellitus, hyperlipidemia, hyperuricemia, and CKD were also documented at enrollment.

\section{Blood sample collection and Inc-MALAT1 determination}

Venous blood samples were collected from AIS patients (within $24 \mathrm{~h}$ after symptoms onset) and controls using ethylene diamine tetraacetic acid (EDTA) tubes, and were subsequently centrifuged at $1600 \mathrm{~g}$ for $10 \mathrm{~min}$ at $4^{\circ} \mathrm{C}$ (within $30 \mathrm{~min}$ ) to acquire supernatant. The supernatant was further centrifuged at $16,000 \mathrm{~g}$ for $10 \mathrm{~min}$ at $4^{\circ} \mathrm{C}$ to obtain the plasma, which was stored at $-80^{\circ} \mathrm{C}$ for further analysis. Inc-MALAT1 relative expression in the plasma of AIS patients and controls was determined by real-time quantitative polymerase chain reaction (RT-qPCR). AIS patients' CRP concentration in plasma was detected using fully automatic POCT fluorescence immunoassay analyzer (Getein Biotech, China), and the plasma level of inflammatory cytokines including tumor necrosis factor $\alpha$ (TNF- $\alpha$ ), interleukin-6 (IL-6), IL-8, IL-10, IL-17, and IL-22 were measured by human enzyme-linked immunosorbent assay (ELISA) kits (Thermo Fisher Scientific, USA) according to the manufacturer's recommendations.

\section{RT-qPCR}

Using QIAamp RNA Blood Mini kit (Qiagen, Germany), total RNA was extracted from plasma samples. Then, reverse transcription to cDNA was performed using PrimeScript $^{\mathrm{TM}}$ RT reagent kit (Perfect Real Time) (Takara, China), and qPCR was performed using TB Green ${ }^{\mathrm{TM}}$ Fast qPCR Mix (Takara). GAPDH was applied as the internal reference. Primers used in the RT-qPCR were as follows: Inc-MALAT1, forward $\left(5^{\prime} \rightarrow 3^{\prime}\right)$ : TCCTAAGGTCAAGAGAA GTGTCAG, reverse $\left(5^{\prime} \rightarrow 3^{\prime}\right)$ : GTGGCGATGTGGCAGA GAA; GAPDH, forward $\left(5^{\prime} \rightarrow 3^{\prime}\right)$ : TGACCACAGTCCATGC CATCAC, reverse $\left(5^{\prime} \rightarrow 3^{\prime}\right)$ : GCCTGCTTCACCACCTTC TTGA.

\section{Follow-up}

All AIS patients received appropriate treatments as recommended by the 2013 AIS Guidelines (AHA/ASA; 13), and were followed-up regularly or as clinically indicated. The last follow-up date was June 30,2018 , and the median follow-up duration was 25.0 months (range: 1.0-42.0 months). RFS was calculated from the date of hospital admission to the date of recurrence or death. 


\section{Statistical analysis}

SPSS 24.0 statistical software (IBM, USA) was used for statistical data processing, and GraphPad Prism 6.01 (GraphPad Software Inc., USA) was applied for graph plotting. Continuous variables are reported as means $\pm S D$ or median and interquartile range (IQR), and the categorical variables are reported as number (percentage). Differences between groups were determined by Student's $t$-test, Wilcoxon rank-sum test, or chi-squared test. Correlation between variables was analyzed by Spearman rank test. Diagnostic value of variables was assessed by receiver operating characteristic (ROC) curve analysis and the derived area under the curve (AUC) as well as $95 \%$ confidence interval $(\mathrm{CI})$. RFS profile is shown by plotting the Kaplan-Meier (K-M) curve, and the difference of RFS between groups was determined by the log-rank test. Factors affecting RFS were analyzed by univariate and multivariate Cox's proportional hazards regression models. All tests were two-sided, and a $\mathrm{P}$ value $<0.05$ was considered statistically significant.

\section{Results}

\section{Characteristics of AIS patients and controls}

No difference of age $(P=0.363)$, gender $(P=0.479)$, and BMI $(P=0.159)$ between AIS patients and controls was observed. Also, the percentage of smoking $(P=0.796)$, hypertension $(P=0.421)$, diabetes mellitus $(P=0.182)$, hyperlipidemia $(P=0.296)$, hyperuricemia $(P=0.888)$, as well as CKD $(P=0.360)$ was similar between the two groups. The detailed information about other baseline characteristics are shown in Table 1.

\section{Disease severity and inflammatory factors in AIS patients}

Mean NIHSS score and mean values of inflammatory factors are reported in Table 2.

\section{Comparison of Inc-MALAT1 expression between AIS patients and controls}

Inc-MALAT1 expression was decreased in AIS patients compared to controls $(P<0.001)$ (Figure 1A). The ROC

Table 1. Characteristics of acute ischemic stroke (AIS) patients and controls.

\begin{tabular}{lccc}
\hline Parameters & AIS patients & Controls & P value \\
\hline Age, years & $62.4 \pm 12.3$ & $61.1 \pm 11.0$ & 0.363 \\
Gender (male/female) & $82 / 38$ & $87 / 33$ & 0.479 \\
BMl, kg/m & $24.6 \pm 2.8$ & $24.1 \pm 2.8$ & 0.159 \\
Smoking & $58(48.3)$ & $60(50.0)$ & 0.796 \\
Hypertension & $108(90.0)$ & $104(86.7)$ & 0.421 \\
Diabetes mellitus & $26(21.7)$ & $18(15.0)$ & 0.182 \\
Hyperlipidemia & $55(45.8)$ & $47(39.2)$ & 0.296 \\
Hyperuricemia & $35(29.2)$ & $36(30.0)$ & 0.888 \\
CKD & $20(16.7)$ & $15(12.5)$ & 0.360 \\
\hline
\end{tabular}

Data are reported as means $\pm S D$ or number (percentage) for $n=120 /$ group. $\mathrm{P}<0.05$ (Student's $t$-test or chi-squared test). BMI: body mass index; CKD: chronic kidney disease.

Table 2. Disease severity, C-reactive protein (CRP), and inflammatory cytokines of acute ischemic stroke patients.

\begin{tabular}{lrrrr}
\hline Parameters & Mean & SD & Median & \multicolumn{1}{c}{ IQR } \\
\hline NIHSS score & 7.8 & 3.5 & 7.0 & $5.0-10.0$ \\
CRP, mg/L & 35.3 & 21.6 & 29.0 & $23.2-38.7$ \\
TNF- $\alpha, \mathrm{pg} / \mathrm{mL}$ & 79.5 & 56.1 & 60.1 & $44.9-94.6$ \\
IL-6, pg/mL & 51.7 & 29.9 & 44.7 & $38.1-54.3$ \\
IL-8, pg/mL & 62.0 & 35.1 & 50.4 & $40.2-76.4$ \\
IL-10, pg/mL & 23.3 & 12.6 & 20.9 & $14.1-30.9$ \\
IL-17, pg/mL & 101.9 & 49.1 & 96.2 & $65.3-122.7$ \\
IL-22, pg/mL & 85.8 & 64.9 & 67.8 & $48.5-96.9$ \\
\hline
\end{tabular}

SD: standard deviation; IQR: inter-quartile range; NIHSS: National Institutes of Health Stroke Scale; TNF- $\alpha$ : tumor necrosis factor $\alpha$; IL: interleukin. 
A

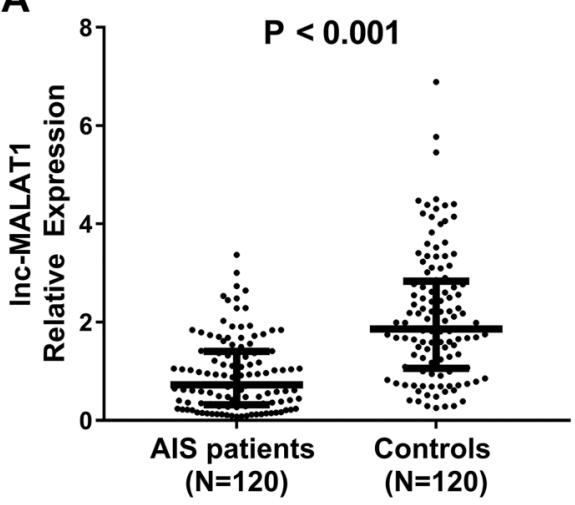

B

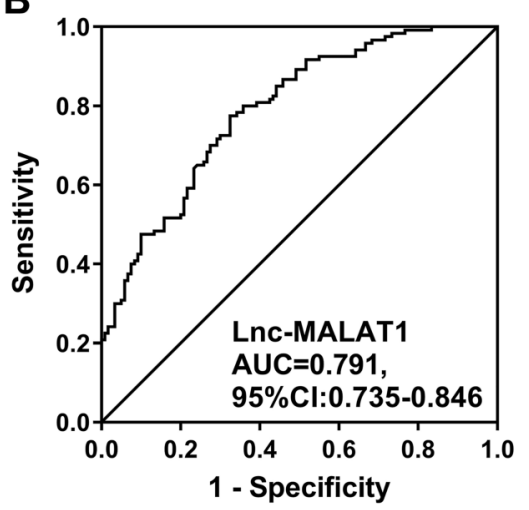

Figure 1. Inc-MALAT1 relative expression in acute ischemic stroke (AIS) patients and controls. Horizontal lines indicate means \pm SD (Wilcoxon rank sum test) (A). Diagnostic value of IncMALAT1 for AIS was assessed by ROC curve (B). Inc-MALAT1: long non-coding RNA metastasis-associated lung adenocarcinoma transcript 1; ROC curve: receiver operating characteristic curve; AUC: area under the curve.

curve showed that Inc-MALAT1 had a close correlation with AIS (AUC=0.791, 95\% Cl: 0.735-0.846) (Figure 1B).

\section{Correlation of Inc-MALAT1 expression with NIHSS score in AIS patients}

Inc-MALAT1 expression was negatively correlated with NIHSS score in AIS patients $(P<0.001, r=-0.437)$ (Figure 2).

\section{Correlation of Inc-MALAT1 expression with inflammatory factors in AIS patients}

Lnc-MALAT1 expression was negatively associated with expression of inflammatory factors including CRP $(P<0.001, r=-0.354)$ (Figure 3A), TNF- $\alpha(P=0.021, r=$ $-0.211)$ (Figure 3B), IL-6 ( $<<0.001, r=0.328)$ (Figure $3 C$ ), IL-8 $(P=0.037, r=-0.191)$ (Figure 3D), and IL-22 ( $P=$ $0.017, r=-0.218$ ) (Figure $3 G$ ), while it was positively correlated with expression of anti-inflammatory cytokine

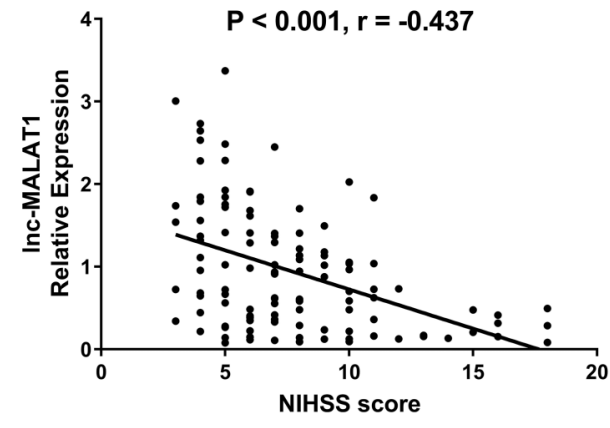

Figure 2. Inc-MALAT1 high expression was correlated with decreased NIHSS score (Spearman rank test). Inc-MALAT1: long non-coding RNA metastasis-associated lung adenocarcinoma transcript 1; NIHSS score: National Institutes of Health Stroke Scale score.

IL-10 ( $P=0.010, r=0.235)$ (Figure 3E) in AIS patients. Furthermore, no correlation of Inc-MALAT1 expression with IL-17 expression ( $P=0.250, r=-0.106)$ (Figure $3 F$ ) was found.

\section{Correlation of Inc-MALAT1 expression with some risk factors for AIS}

To investigate the correlation of Inc-MALAT1 expression with some risk factors for AIS, all patients were categorized into different subgroups according to the risk factors (Figure 4). Lnc-MALAT1 expression was elevated in diabetes compared to non-diabetes $(P=0.017)$ (Figure 4B). Notably, although no difference of IncMALAT1 expression with hypertension was observed, there was a trend for reduced hypertension in Inc-MALAT1 high expression patients compared to Inc-MALAT1 low expression ( $P=0.081$ ) (Figure 4A). No difference of Inc-MALAT1 expression was found between hyperlipidemia patients and non-hyperlipidemia patients $(P=0.391)$ (Figure 4C), hyperuricemia patients and non-hyperuricemia patients $(P=0.657)$ (Figure 4D), or CKD patients and non-CKD patients $(P=0.264)$ (Figure 4E). These data indicated that Inc-MALAT1 expression was positively correlated with diabetes occurrence in AIS patients. Additionally, no correlation of Inc-MALAT1 expression with risk factors including hypertension $(P=0.757)$, diabetes mellitus $(P=$ $0.082)$, hyperlipidemia $(P=0.232)$, hyperuricemia $(P=0.740)$, and CKD ( $P=0.642)$ was found in the control group (Supplementary Table S1). However, a trend for increased Inc-MALAT1 expression was observed in diabetes mellitus patients compared to non-diabetes mellitus patients in the control group.

\section{Comparison of RFS between Inc-MALAT1 high expression patients and Inc-MALAT1 low expression patients}

Patients were divided into Inc-MALAT1 high expression group and Inc-MALAT1 low expression group according to 
A

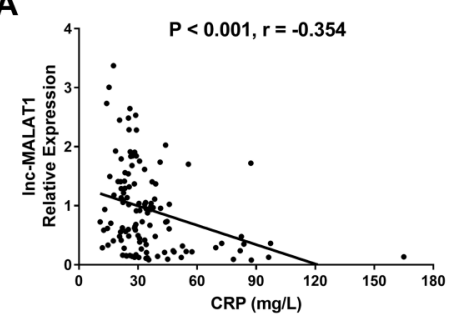

C

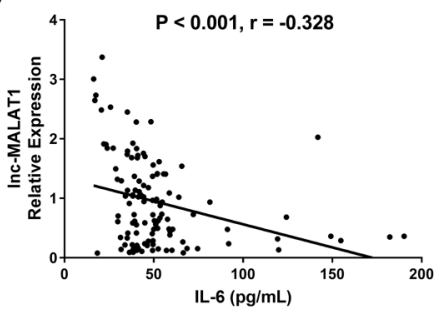

E

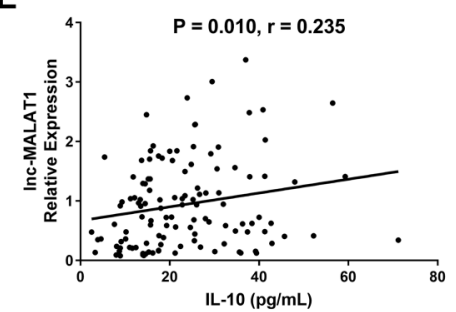

$\mathbf{G}$

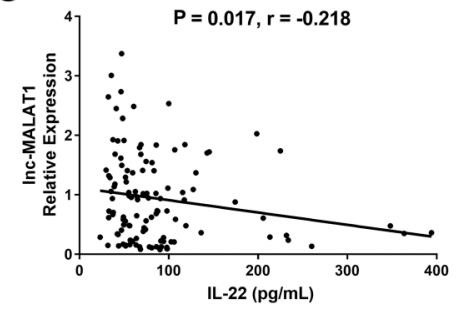

B

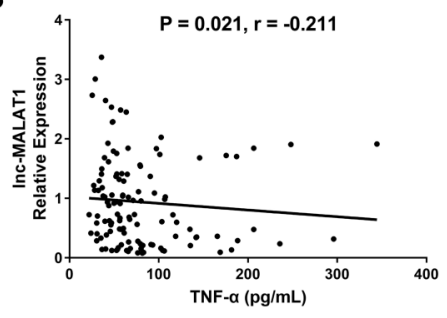

D

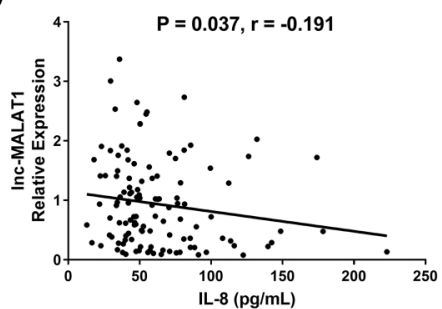

$\mathbf{F}$

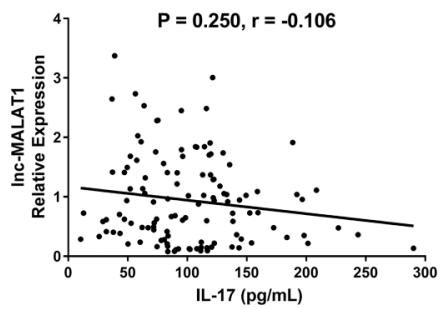

Figure 3. Correlation of Inc-MALAT1 expression with C-reactive protein (CRP) (A), tumor necrosis factor $\alpha$ (TNF- $\alpha$ ) (B), interleukin (IL)-6 (C), IL-8 (D), IL-10 (E), IL-17 (F), and IL-22 (G) was determined by the Spearman rank test. Inc-MALAT1: long non-coding RNA metastasis-associated lung adenocarcinoma transcript 1.

the median value of Inc-MALAT1 expression. No difference of RFS was found between the groups, while there was a trend for longer RFS in Inc-MALAT1 high expression ( $P=$ 0.053) compared to Inc-MALAT1 low expression (Figure 5).

\section{Analysis of factors affecting RFS in AIS patients}

Univariate Cox's regression analysis showed that there was a trend for better RFS in Inc-MALAT1 high expression patients compared to Inc-MALAT1 low expression patients (although statistically non-significant) $(\mathrm{P}=$ 0.063 ), while CRP ( $\geqslant 29.0$ vs $<29.0 \mathrm{mg} / \mathrm{L})$ was associated with worse RFS ( $P=0.006)$ (Table 3$)$. Moreover, multivariate Cox's regression analysis showed that IncMALAT1 high expression was not an independent predictive factor for RFS, whereas CRP ( $\geqslant 29.0$ vs $<29.0 \mathrm{mg} / \mathrm{L})$ independently predicted reduced RFS ( $P=0.024)$.

\section{Discussion}

Our results indicated that: 1) Inc-MALAT1 expression was decreased in AIS patients compared to controls, and it had a close correlation with AIS; 2) Inc-MALAT1 high expression was correlated with decreased NIHSS score as well as reduced inflammatory factors levels in AIS patients; and 3) Inc-MALAT1 high expression showed a trend for prolonged RFS compared to Inc-MALAT1 low expression in AIS patients, but without statistical significance. 
A

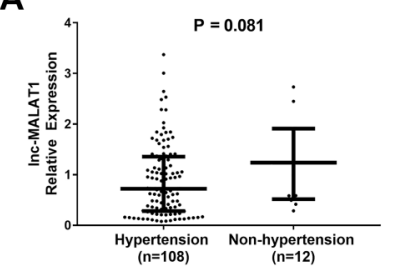

D

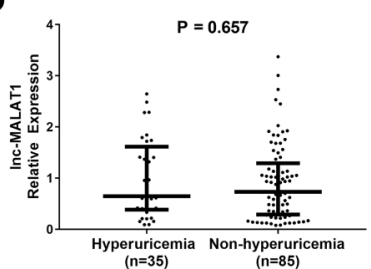

B

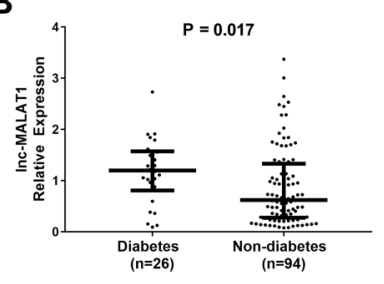

E

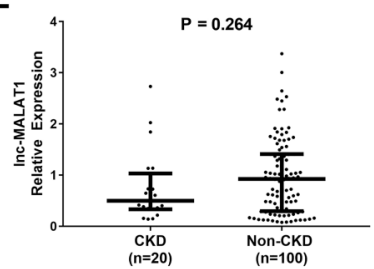

C

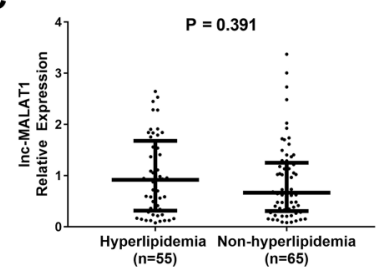

Figure 4. Inc-MALAT1 expression in acute ischemic stroke patients with different risk factors: hypertension and non-hypertension patients (A), diabetes and non-diabetes patients (B), hyperlipidemia and non-hyperlipidemia patients (C), hyperuricemia and non-hyperuricemia patients (D), as well as chronic kidney disease (CKD) and non-CKD patients (E). Horizontal lines indicate means \pm SD (Wilcoxon rank sum test). Inc-MALAT1: long non-coding RNA metastasis-associated lung adenocarcinoma transcript 1.

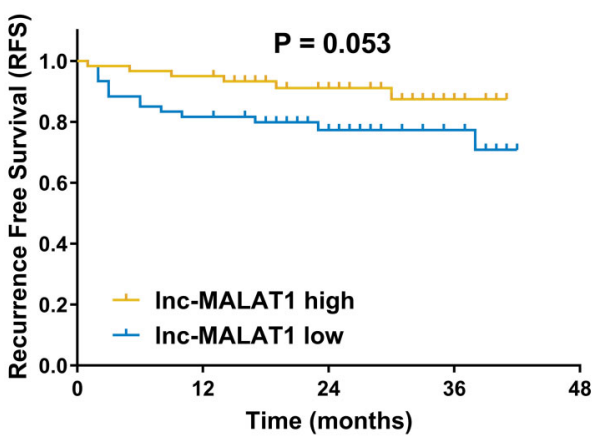

Figure 5. Kaplan-Meier curves show the recurrence-free survival in Inc-MALAT1 high expression and Inc-MALAT1 low expression patients determined by the log-rank test. Inc-MALAT1: long noncoding RNA metastasis-associated lung adenocarcinoma transcript 1.

IncRNAs are involved in a variety of biological processes (such as gene transcription, organizing RNAprotein complex, and management in protein activity) (16-19). As one of the frequently investigated IncRNAs, Inc-MALAT1 has various molecular functions (including alternative splicing, transcriptional regulation, and posttranscriptional regulation), and also participates in multiple physiological functions (including neural development, skeletal myogenesis, and vascular growth) (7). As to its role in neurological diseases or cerebrovascular diseases, previous studies demonstrate that Inc-MALAT1 presents protective effects in these diseases via repressing proapoptotic or pro-inflammatory factors $(10,11,20,21)$. For example, one study reported that Inc-MALAT1 protects human brain vascular endothelial cells from OGD-induced apoptosis through activating phosphatidylinositol 3-kinase (PI3K) (21). In addition, Inc-MALAT1 expression is decreased in the spinal cords of mice with experimental autoimmune encephalomyelitis, and its knockdown raises the level of inflammatory cytokines (including IL-1 and IL-6) (11). Also, silencing of Inc-MALAT1 raises the level of pro-apoptotic factor Bim and pro-inflammatory cytokines (including MCP-1 as well as E-selectin) in BMECs following oxygen-glucose deprivation (OGD), which is an in vitro mimic of ischemic stroke conditions (10). Another study discloses that Inc-MALAT1 knockdown aggravates OGD-induced overexpression of pro-inflammatory cytokines including MCP-1 and IL- 6 in mouse cerebral microvascular endothelial cells (20). These studies reveal that Inc-MALAT1 has a protective effect in neurological and cerebrovascular diseases through reducing inflammation or inhibiting cell apoptosis.

Previous clinical trials focus mainly on the exploration of Inc-MALAT1 in cancer patients, showing that IncMALAT1 plays a tumor-promotive role, while information about the role of Inc-MALAT1 in neurological diseases or cerebrovascular diseases is still limited (22-24). Only a few studies show that Inc-MALAT1 expression is reduced in central nervous system tissues from multiple sclerosis patients and carotid plaques from atherosclerosis patients $(11,12)$. As for the correlation of Inc-MALAT1 with disease severity and inflammation in neurological and cerebrovascular disease patients, only one study shows that elevated Inc-MALAT1 expression is correlated with less advanced lesions in atherosclerosis patients (12). Considering that Inc-MALAT1 might participate in the cerebrovascular pathologies of stroke according to previous studies, and it presented an anti-inflammatory effect in cardiovascular 
Table 3. Factors affecting recurrence-free survival by Cox's proportional hazards regression model analyses.

\begin{tabular}{|c|c|c|c|c|}
\hline \multirow[t]{2}{*}{ Items } & \multicolumn{2}{|c|}{ Univariate Cox's regression } & \multicolumn{2}{|c|}{ Multivariate Cox's regression } \\
\hline & $P$ value & $\mathrm{HR}(95 \% \mathrm{Cl})$ & $P$ value & $\mathrm{HR}(95 \% \mathrm{Cl})$ \\
\hline Inc-MALAT1 (high vs low) & 0.062 & $0.402(0.154-1.047)$ & 0.200 & $0.482(0.157-1.473)$ \\
\hline Age $(\geqslant 61$ vs $<61$ years $)$ & 0.976 & $0.986(0.406-2.397)$ & 0.974 & $0.981(0.307-3.137)$ \\
\hline Gender (male/female) & 0.853 & $1.095(0.421-2.850)$ & 0.965 & $0.974(0.304-3.120)$ \\
\hline $\mathrm{BMI}\left(\geqslant 24.2\right.$ vs $\left.<24.2 \mathrm{~kg} / \mathrm{m}^{2}\right)$ & 0.506 & $0.741(0.307-1.791)$ & 0.725 & $0.835(0.305-2.282)$ \\
\hline Smoking (yes vs no) & 0.456 & $0.712(0.291-1.741)$ & 0.509 & $0.726(0.281-1.876)$ \\
\hline Hypertension (yes vs no) & 0.836 & $1.167(0.270-5.047)$ & 0.704 & $1.371(0.269-6.980)$ \\
\hline Diabetes mellitus (yes vs no) & 0.193 & $0.378(0.088-1.633)$ & 0.526 & $0.585(0.111-3.075)$ \\
\hline Hyperlipidemia (yes vs no) & 0.853 & $0.920(0.380-2.227)$ & 0.978 & $1.015(0.365-2.821)$ \\
\hline Hyperuricemia (yes vs no) & 0.958 & $1.026(0.394-2.673)$ & 0.712 & $0.819(0.283-2.370)$ \\
\hline CKD (yes vs no) & 0.821 & $1.136(0.377-3.418)$ & 0.609 & $1.435(0.360-5.728)$ \\
\hline NIHSS score $(\geqslant 7$ vs $<7)$ & 0.428 & $0.701(0.292-1.687)$ & 0.229 & $0.557(0.215-1.445)$ \\
\hline $\mathrm{CRP}(\geqslant 29.0$ vs $<29.0 \mathrm{mg} / \mathrm{L})$ & 0.006 & $4.683(1.563-14.028)$ & 0.024 & $4.720(1.224-18.197)$ \\
\hline TNF- $\alpha(\geqslant 60.1 v s<60.1 \mathrm{pg} / \mathrm{mL})$ & 0.126 & $2.052(0.818-5.151)$ & 0.350 & $1.879(0.501-7.041)$ \\
\hline IL-6 ( $\geqslant 44.7$ vs $<44.7 \mathrm{pg} / \mathrm{mL})$ & 0.301 & $1.605(0.655-3.931)$ & 0.899 & $1.069(0.381-2.996)$ \\
\hline $\mathrm{IL}-8(\geqslant 50.4$ vs $<50.4 \mathrm{pg} / \mathrm{mL})$ & 0.988 & $0.993(0.413-2.387)$ & 0.387 & $0.559(0.149-2.088)$ \\
\hline $\mathrm{IL}-10(\geqslant 20.9$ vs $<20.9 \mathrm{pg} / \mathrm{mL})$ & 0.133 & $0.493(0.196-1.239)$ & 0.548 & $0.707(0.227-2.195)$ \\
\hline $\mathrm{IL}-17(\geqslant 96.2$ vs $96.2 \mathrm{pg} / \mathrm{mL})$ & 0.586 & $0.783(0.324-1.889)$ & 0.153 & $0.474(0.170-1.319)$ \\
\hline $\mathrm{IL}-22(\geqslant 67.8$ vs $<67.8 \mathrm{pg} / \mathrm{mL})$ & 0.299 & $1.608(0.656-3.942)$ & 0.716 & $0.797(0.235-2.705)$ \\
\hline
\end{tabular}

HR: hazard ratio; Cl: confidence interval; BMI: body mass index; CKD: chronic kidney disease; NIHSS: National Institutes of Health Stroke Scale; CRP: C-reactive protein; TNF- $\alpha$ : tumor necrosis factor $\alpha$; IL: interleukin. Significant P values are shown in bold.

and cerebrovascular diseases, we hypothesized that IncMALAT1 expression might be related to disease risk, progression, or inflammation level of AIS (9-12,25).

In our study, we assessed the association of IncMALAT1 with AIS, and observed that Inc-MALAT1 expression was lower in AIS patients compared to controls, and Inc-MALAT1 expression presented good diagnostic value for AIS, which might be due to its protective effect via repressing cell apoptosis of BMECs in these diseases. Moreover, we investigated the correlation of Inc-MALAT1 expression with disease severity as well as inflammation in AIS patients. We observed that Inc-MALAT1 high expression was associated with decreased NIHSS score and reduced level of inflammatory factors (including CRP, TNF- $\alpha$, IL-6, IL-8, and IL-22). In addition, there was a trend for an increased proportion of diabetes (without statistical significance) in AIS patients with IncMALAT1 high expression. The possible reasons for these results might be as follows: 1 ) Inc-MALAT1 had a protective effect on brain microvascular endothelial cells through inhibiting cell apoptosis, thereby contributed to maintaining healthy brain endothelium that was essential for normal cerebrovascular physiology, therefore the severity of AIS was attenuated and a decreased NIHSS score was observed $(7,20) ; 2$ ) Inc-MALAT1 might decrease the expression of transcriptional factor nuclear factor-kappa $\mathrm{B}(\mathrm{NF}-\kappa \mathrm{B})$ that drove the transcription of a series of inflammatory factors, thus led to reduced inflammatory factor level and alleviated inflammation in AIS patients (26); 3) Inc-MALAT1 protected the brain microvascular endothelial cells against apoptosis, thereby facilitated microvascular permeability and helped restore cerebral vasoreactivity, thus it might attenuate hypertension in AIS patients (25); and 4) Inc-MALAT1 was found to interact with transcription factor Foxo1 and SIRT1 transcription to induce poor glycemic control and insulin resistance, which might promote the occurrence of diabetes $(27,28)$.

As to the prognostic value of Inc-MALAT1 in human diseases, it has been identified as a valuable biomarker for disease prognosis in several cancers, while limited clinical studies have been found in AIS. Only one study reports that Inc-MALAT1 high expression is associated with prolonged main adverse cardiovascular and cerebrovascular events (MACCE)-free survival in atherosclerosis patients (12). To our knowledge, there is still no evidence about the influence of Inc-MALAT1 on the prognosis in AIS patients. In our study, we observed that there was a trend for better RFS in Inc-MALAT1 high expression AIS patients, but without statistical significance. These results might be due to: 1) Inc-MALAT1 reduced the production of inflammation cytokines, further decreased inflammation, and attenuated disease progression, thus it led to better RFS in AIS patients $(10,20) ; 2)$ Inc-MALAT1 might facilitate sensitivity to treatment in AIS, thereby it increased treatment efficacy and resulted in longer RFS in AIS patients. 
Some limitations existed in our study: 1) the sample size (120 AIS patients) was relatively small, thus the statistical power might be low; 2) the median follow-up duration (25.0 months (range: 1.0-42.0 months)) was relatively short, thus the correlation of Inc-MALAT1 expression with long-term prognosis remains to be further investigated; and 3) detailed mechanism of Inc-MALAT1 in AIS is still unclear, and further study is still needed.

In conclusion, Inc-MALAT1 was downregulated and presented a close association with AIS, and its high

\section{References}

1. Li Y, Zhong W, Jiang Z, Tang X. New progress in the approaches for blood-brain barrier protection in acute ischemic stroke. Brain Res Bull 2019; 144: 46-57, doi: 10.1016/ j.brainresbull.2018.11.006.

2. Heit JJ, Zaharchuk G, Wintermark M. Advanced neuroimaging of acute ischemic stroke: penumbra and collateral assessment. Neuroimaging Clin N Am 2018; 28: 585-597, doi: 10.1016/j.nic.2018.06.004.

3. Wu C, Wu D, Chen J, Li C, Ji X. Why not intravenous thrombolysis in patients with recurrent stroke within 3 months? Aging Dis 2018; 9: 309-316, doi: 10.14336/ AD.2017.0406

4. Luo Y, Tang H, Li H, Zhao R, Huang Q, Liu J. Recent advances in the development of neuroprotective agents and therapeutic targets in the treatment of cerebral ischemia. Eur J Med Chem 2019; 162: 132-146, doi: 10.1016/j.ejmech. 2018.11.014.

5. Sun MS, Jin H, Sun X, Huang S, Zhang FL, Guo ZN, et al. Free radical damage in ischemia-reperfusion injury: an obstacle in acute ischemic stroke after revascularization therapy. Oxid Med Cell Longev 2018; 2018: 3804979, doi: 10.1155/2018/3804979.

6. Leiva-Salinas C, Patrie JT, Xin W, Michel P, Jovin T, Wintermark M. Prediction of early arterial recanalization and tissue fate in the selection of patients with the greatest potential to benefit from intravenous tissue-type plasminogen activator. Stroke 2016; 47: 397-403, doi: 10.1161/ STROKEAHA.115.011066.

7. Zhang $\mathrm{X}$, Hamblin $\mathrm{MH}$, Yin KJ. The long noncoding RNA Malat1: Its physiological and pathophysiological functions. RNA Biol 2017; 14: 1705-1714, doi: 10.1080/15476286. 2017.1358347.

8. Ren W, Yang X. Pathophysiology of Long non-coding RNAs in ischemic stroke. Front Mol Neurosci 2018; 11: 96, doi: 10.3389/fnmol.2018.00096.

9. Yang H, Xi X, Zhao B, Su Z, Wang Z. KLF4 protects brain microvascular endothelial cells from ischemic stroke induced apoptosis by transcriptionally activating MALAT1. Biochem Biophys Res Commun 2018; 495: 2376-2382, doi: 10.1016/ j.bbrc.2017.11.205.

10. Zhang $X$, Tang $X$, Liu K, Hamblin MH, Yin KJ. Long noncoding RNA Malat1 regulates cerebrovascular pathologies in ischemic stroke. J Neurosci 2017; 37: 1797-1806, doi: 10.1523/JNEUROSCI.3389-16.2017.

11. Masoumi F, Ghorbani S, Talebi F, Branton WG, Rajaei S, Power $\mathrm{C}$, et al. Malat1 long noncoding RNA regulates expression correlated with decreased NIHSS score as well as reduced inflammation in AIS patients. Moreover, there was a trend for better RFS in AIS patients with IncMALAT1 high expression compared to those with IncMALAT1 low expression, despite the lack of statistical correlation.

\section{Supplementary Material}

Click here to view [pdf]. inflammation and leukocyte differentiation in experimental autoimmune encephalomyelitis. J Neuroimmunol 2019; 328: 50-59, doi: 10.1016/j.jneuroim.2018.11.013.

12. Cremer S, Michalik KM, Fischer A, Pfisterer L, Jae N, Winter $C$, et al. Hematopoietic deficiency of the long noncoding RNA MALAT1 promotes atherosclerosis and plaque inflammation. Circulation 2019; 139: 1320-1334, doi: 10.1161/ CIRCULATIONAHA.117.029015.

13. Jauch EC, Saver JL, Adams HP Jr, Bruno A, Connors JJ, Demaerschalk BM, et al. Guidelines for the early management of patients with acute ischemic stroke: a guideline for healthcare professionals from the American Heart Association/American Stroke Association. Stroke 2013; 44: 870947, doi: 10.1161/STR.0b013e318284056a.

14. Mi T, Sun S, Zhang G, Carora Y, Du Y, Guo S, et al. Relationship between dyslipidemia and carotid plaques in a high-stroke-risk population in Shandong Province, China. Brain Behav 2016; 6: e00473, doi: 10.1002/brb3.473.

15. Bartakova V, Kuricova K, Pacal L, Nova Z, Dvorakova V, Svrckova M, et al. Hyperuricemia contributes to the faster progression of diabetic kidney disease in type 2 diabetes mellitus. J Diabetes Complications 2016; 30: 1300-1307, doi: 10.1016/j.jdiacomp.2016.06.002.

16. Chen H, Wang X, Yan X, Cheng X, He X, Zheng W. LncRNA MALAT1 regulates sepsis-induced cardiac inflammation and dysfunction via interaction with miR-125b and p38 MAPK/ NFkappaB. Int Immunopharmacol 2018; 55: 69-76, doi: 10.1016/j.intimp.2017.11.038.

17. Dai L, Zhang G, Cheng Z, Wang X, Jia L, Jing X, et al. Knockdown of LncRNA MALAT1 contributes to the suppression of inflammatory responses by up-regulating miR-146a in LPS-induced acute lung injury. Connect Tissue Res 2018; 59: 581-592, doi: 10.1080/03008207.2018.1439480.

18. Yu Z, Rayile A, Zhang X, Li Y, Zhao Q. Ulinastatin protects against lipopolysaccharide-induced cardiac microvascular endothelial cell dysfunction via downregulation of IncRNA MALAT1 and EZH2 in sepsis. Int J Mol Med 2017; 39: 12691276, doi: 10.3892/ijmm.2017.2920.

19. Brock M, Schuoler C, Leuenberger C, Buhlmann C, Haider TJ, Vogel J, et al. Analysis of hypoxia-induced noncoding RNAs reveals metastasis-associated lung adenocarcinoma transcript 1 as an important regulator of vascular smooth muscle cell proliferation. Exp Biol Med (Maywood) 2017; 242: 487-496, doi: 10.1177/1535370216685434.

20. Zhang J, Yuan L, Zhang $X$, Hamblin MH, Zhu T, Meng F, et al. Altered long non-coding RNA transcriptomic profiles in 
brain microvascular endothelium after cerebral ischemia. Exp Neurol 2016; 277: 162-170, doi: 10.1016/j.expneurol. 2015.12.014.

21. Xin JW, Jiang YG. Long noncoding RNA MALAT1 inhibits apoptosis induced by oxygen-glucose deprivation and reoxygenation in human brain microvascular endothelial cells. Exp Ther Med 2017; 13: 1225-1234, doi: 10.3892/etm. 2017.4095.

22. Arun G, Spector DL. MALAT1 long non-coding RNA and breast cancer. RNA Biol 2019; 16: 860-863, doi: 10.1080/ 15476286.2019.1592072.

23. Zhuang $M$, Zhao $S$, Jiang Z, Wang $S$, Sun $P$, Quan J, et al. MALAT1 sponges miR-106b-5p to promote the invasion and metastasis of colorectal cancer via SLAIN2 enhanced microtubules mobility. EBioMedicine 2019; 41: 286-298, doi: 10.1016/j.ebiom.2018.12.049.

24. Li Z, Xu L, Liu Y, Fu S, Tu J, Hu Y, et al. LncRNA MALAT1 promotes relapse of breast cancer patients with postoperative fever. Am J Transl Res 2018; 10: 3186-3197.
25. Vitt JR, Trillanes M, Hemphill JC 3rd. Management of blood pressure during and after recanalization therapy for acute ischemic stroke. Front Neurol 2019; 10: 138, doi: 10.3389/ fneur.2019.00138.

26. Tian $\mathrm{H}$, Wu M, Zhou $\mathrm{P}$, Huang $\mathrm{C}$, Ye $\mathrm{C}$, Wang $\mathrm{L}$. The long non-coding RNA MALAT1 is increased in renal ischemiareperfusion injury and inhibits hypoxia-induced inflammation. Renal failure 2018; 40: 527-533, doi: 10.1080/08860 22X.2018.1487863.

27. Sathishkumar C, Prabu P, Mohan V, Balasubramanyam M. Linking a role of IncRNAs (long non-coding RNAs) with insulin resistance, accelerated senescence, and inflammation in patients with type 2 diabetes. Hum Genomics 2018; 12: 41, doi: 10.1186/s40246-018-0173-3.

28. Zhou L, Xu DY, Sha WG, Shen L, Lu GY. Long non-coding RNA MALAT1 interacts with transcription factor Foxo1 to regulate SIRT1 transcription in high glucose-induced HK2cells injury. Biochem Biophys Res Commun 2018; 503: 849-855, doi: 10.1016/j.bbrc.2018.06.086. 